\title{
Evaluating the potential impact of an emergency department extracorporeal resuscitation (ECPR) program: a health records review
}

\author{
Laura McDonald, BHSc ${ }^{*}$; George Mastoras ${ }^{(0,}, \mathrm{MD}^{\dagger}$; Michael Hickey, $\mathrm{MD}^{\dagger,}$; \\ Bernard McDonald, MD, $\mathrm{PhD}^{\S}$; Edmund S.H. Kwok (D), MD, MHA ${ }^{\dagger}$
}

\begin{abstract}
CLINICIAN'S CAPSULE
What is known about the topic?

Extracorporeal cardiopulmonary resuscitation (ECPR) is an emerging resuscitative modality that could improve survival for ED patients with refractory cardiac arrest.

What did this study ask?

What proportion of refractory cardiac arrests would be eligible for ECPR at our center, based on published inclusion/exclusion criteria?

What did this study find?

This 2-year health records review found that a small but meaningful number of ED patients could be eligible for ECPR.

Why does this study matter to clinicians?

Institutions need a clear picture of the potential impact for ED and hospital operations before initiation of ECPR programs.
\end{abstract}

\section{ABSTRACT}

Objectives: Extracorporeal cardiopulmonary resuscitation in refractory cardiac arrest (ECPR) is an emerging resuscitative therapy that has shown promising results for selected patients who may not otherwise survive. We sought to identify the characteristics of cardiac arrest patients presenting to our institution to begin assessing the feasibility of an ECPR program.

Methods: This retrospective health records review included patients aged 18-75 years old presenting to our academic teaching hospital campuses with refractory nontraumatic out-of-hospital or in-emergency department (ED) cardiac arrest over a 2-year period. Based on a scoping review of the literature, both "liberal" and "restrictive" ECPR criteria were defined and applied to our cohort.
Results: A total of 179 patients met inclusion criteria. Median age was 60 years, and patients were predominantly male $(72.6 \%)$. The initial rhythm was ventricular tachycardia/ventricular fibrillation in $49.2 \%$. The majority of arrests were witnessed $(69.3 \%)$, with immediate bystander CPR performed on $53.1 \%$ and an additional $12 \%$ receiving CPR within 10 minutes of collapse. Median prehospital time was 40 minutes (interquartile range, 31-53.3). Two-thirds of patients $(65.9 \%)$ were identified as having a reversible cause of arrest and favorable premorbid status was identified in nearly three quarters (74.3\%). Our two sets of ECPR inclusion criteria revealed that 33 and 5 patients (liberal and restrictive criteria, respectively), would have been candidates for ECPR.

Conclusion: At our institution, we estimate between $6 \%$ and $40 \%$ of ED refractory cardiac arrest patients would be candidates for ECPR. These findings suggest that the implementation of an ECPR program should be explored.

\section{RÉSUMÉ}

Introduction: La réanimation extracorporelle (REC) dans les cas d'arrêt cardiaque réfractaire est une toute nouvelle technique de réanimation qui a donné des résultats prometteurs chez certains patients qui autrement n'auraient peut-être pas survécu à l'événement. L'étude visait donc à cerner les caractéristiques des patients ayant subi un arrêt cardiaque et ayant été transportés dans l'établissement des auteurs, et à évaluer la faisabilité d'un programme de REC.

Méthode: II s'agit d'un examen rétrospectif de dossiers médicaux de patients âgés de 18 à 75 ans, qui ont été transportés à I'hôpital d'enseignement désigné et qui avaient subi, sur une période de 2 ans, un arrêt cardiaque réfractaire, d'origine non traumatique, en milieu extrahospitalier ou au service des urgences (SU). Après un examen de délimitation de la

From the *Faculty of Medicine, University of Ottawa, Ottawa, ON; ${ }^{\dagger}$ Department of Emergency Medicine, Faculty of Medicine, University of Ottawa, Ottawa, ON; ${ }^{\ddagger}$ Division of Critical Care, Department of Medicine, Faculty of Medicine, University of Ottawa, Ottawa, ON; and the ${ }^{\S}$ Division of Cardiac Anesthesia, Department of Anesthesiology and Pain Medicine, University of Ottawa, Ottawa, ON.

Correspondence to: Dr. George Mastoras, 1053 Carling Avenue, E-Main, Room EM-206, Box 227, The Ottawa Hospital, Civic Campus, Ottawa, Ontario, Canada. K1Y 4E; Email: george.mastoras@gmail.com

(c) Canadian Association of Emergency Physicians 2020

CJEM 2020;22(3):375-378

DOI 10.1017/cem.2019.472 
documentation, des critères " élargis " et des critères " restrictifs " de REC ont d'abord été établis, puis appliqués à la cohorte de patients.

Résultats: Au total, 179 patients respectaient les critères de sélection. L'âge médian était de 60 ans, et les sujets étaient en grande partie des hommes (72,6\%). Dans $49,2 \%$ des cas, le rythme initial était de la TV ou de la FV. La majorité des arrêts cardiaques se sont produits en présence de témoins $(69,3 \%)$; dans $53,1 \%$ des cas, la RCR a été pratiquée immédiatement par des spectateurs et, dans $12 \%$ de cas supplémentaires, dans les 10 minutes suivant l'effondrement de la personne. Le temps médian avant l'arrivée à I'hôpital était de 40 minutes (intervalle interquartile : 31-53,3). Une cause réversible d'arrêt cardiaque a été mise en évidence chez les deux tiers des patients $(65,9 \%)$ et un état morbide favorable préexistant, chez presque les trois quarts $(74,3 \%)$ des patients. Enfin, 33 sujets et 5 sujets auraient été candidats à la REC, selon le type de critères appliqués (élargis ou restrictifs, respectivement).

Conclusion: De $6 \%$ à $40 \%$ des patients traités pour un arrêt cardiaque réfractaire au SU auraient été candidats à la REC, dans l'établissement où a été réalisée l'étude. Les résultats obtenus donnent à penser qu'il serait justifié d'examiner la mise sur pied d'un programme de REC.

Keywords: Cardiac arrest, resuscitation, critical care

\section{INTRODUCTION}

Cardiac arrests have historically poor outcomes. For out-of-hospital cardiac arrest, the inevitable delay to appropriate treatment and limitations of conventional treatment results in survival rates of less than $10 \% .^{1-4}$ However, a novel resuscitative therapy known as extracorporeal cardiopulmonary resuscitation (ECPR) has recently shown promising results in patients with refractory cardiac arrest., ${ }^{4,5}$ ECPR is a form of extracorporeal cardiopulmonary support used to resuscitate the failing circulatory system in cases of refractory cardiac arrest or cardiogenic shock. While still in its infancy, the use of ECPR has been described in numerous centers with impressive results in appropriately selected patients; namely, neurologically intact survival in excess of $30 \%$ for refractory out-of-hospital cardiac arrest. ${ }^{4-6}$ Despite this widespread interest, there exists many barriers to the implementation of what can be considered a low volume and resource-intensive endeavor. Therefore, the aim of this study is to assess the potential impact of an ECPR program in the context of a high-volume, tertiary care, academic emergency department that currently partners with cardiac care services in a regionalized ST-elevation myocardial infarction (STEMI) and post-return of spontaneous circulation (ROSC) care program. Specifically, we sought to retrospectively review the characteristics of cardiac arrest patients who presented to our ED and assess if they would meet criteria for ECPR according to current literature. ${ }^{5}$

\section{METHODS}

\section{Study design and settings}

This is a retrospective health records review of selected patients who presented with out-of-hospital cardiac arrest or ROSC to either campus of our tertiary-care academic hospital during a 2-year period from January 1, 2015, to December 31, 2016. This investigation was approved by the Ottawa Health Science Network Research Ethics Board and conducted at The Ottawa Hospital.

\section{Selection of participants}

We abstracted data from the health records of all patients aged 18 to 75 years within the defined 2-year time period who presented to our ED with a chief complaint of cardiac arrest, vital signs absent, or ROSC, or an ED discharge diagnosis of cardiac arrest, ROSC, or death. Based on our review of existing ECPR literature, we created an extensive list of characteristics to extract from charts that could be used to extrapolate relevant prognostic information. Exclusion criteria included: traumatic cardiac arrest etiology, no true arresting rhythm identified at any point, and transfers to our institution from a peripheral ED.

\section{Establishment of ECPR criteria}

We performed a scoping review of existing ECPR literature to define a priori two sets of liberal and restrictive 


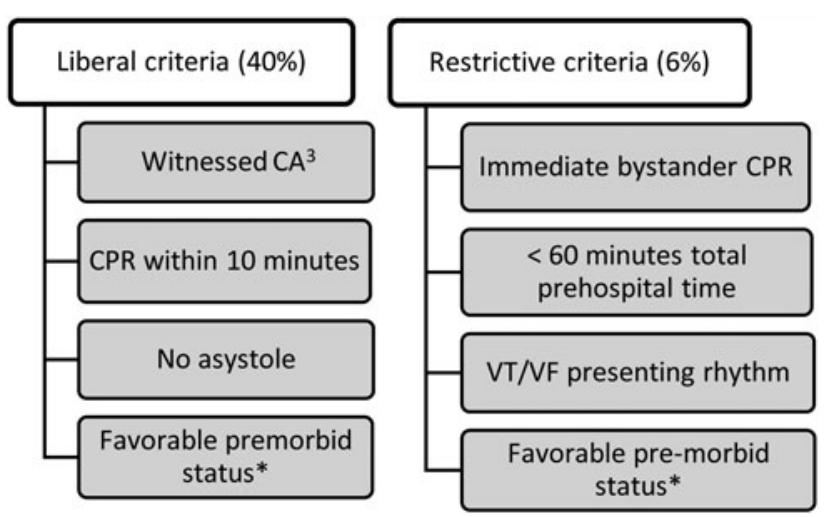

Figure 1. ECPR eligibility criteria. * Favorable pre-morbid status was defined as an absence of significant pre-arrest morbidities, including severe chronic obstructive pulmonary disease (COPD), severe heart disease, dependency, life expectancy $<1$ year, and terminal cancer. Note: Reported percentages reference proportion of refractory out-of-hospital cardiac arrest that would have been ECPR-eligible by these criteria. CA, cardiac arrest; VT/VF, ventricular tachycardia/ ventricular fibrillation.

criteria to later apply to our cohort for possible initiation of ECPR. These criteria (see Figure 1), were selected based on either their association with survival and good neurological outcome, or their recurring use as inclusion/exclusion criteria in the ECPR literature. Witnessed cardiac arrest, CPR within 10 minutes, ${ }^{5-7}$ immediate bystander $\mathrm{CPR}^{4}$ and $<60$ minutes prehospital time were shown to be predictors of good outcome ( $p$ value $\leq 0.05)$.

A shockable recorded cardiac arrest rhythm was selected based on the findings of Yannopoulos et al., ${ }^{5}$ who used only patients meeting these criteria for their observational study, citing the overwhelming majority $(>80 \%)$ of cardiac arrest survivors emerging from this group. ${ }^{5}$ Nonasystole rhythms have been described in other studies ${ }^{4}$ and may have a role in more liberal extracorporeal life support programs (i.e., massive pulmonary embolism).

The presence of a major pre-existing medical co-morbidity was an almost universally used exclusion criteria in the ECPR literature. ${ }^{6,9,10}$ As such, the absence of such pre-existing co-morbidities, including severe COPD, severe heart disease, functional dependency, life expectancy $<1$ year, and terminal cancer, was selected as both a liberal and restrictive criteria under "favorable premorbid status."

The categorization of these criteria as either "liberal" or "restrictive," was done by determining which criteria corresponded to higher or lower numbers of cardiac arrest cases, based on the results of the scoping review. ${ }^{5-7}$

\section{Data collection and quality control}

Charts were manually reviewed using our hospital electronic medical record which archives lab data, diagnostic imaging, scans of handwritten paper charts and dictated notes. The chart review was conducted by one of the principal investigators (L.M.), with $20 \%$ of charts randomly reviewed by a second investigator (G.M.) to ensure calibration. Any discrepancies or ambiguities found in the review were resolved by consensus between both investigators.

\section{Analysis}

Data were deidentified and entered into a spreadsheet (Microsoft Excel, 2013). Simple descriptive statistics were performed to identify eligible candidates.

\section{RESULTS}

During the study period, 220 patients with out-of-hospital cardiac arrest in the selected age group were brought to our EDs (Supplemental Online Appendix B). A total of 33 patients were excluded from the study based on criteria identified above: 32 for traumatic cardiac arrest, and 1 patient who was determined to have never suffered an arresting rhythm. A further 105 patients achieved ROSC. Characteristics of all out-of-hospital cardiac arrest patients included in the analysis are described in the Supplemental Online Appendix A.

We determined that of the remaining 82 patients with refractory out-of-hospital cardiac arrest, all of whom died, two-thirds $(65.9 \%)$ had favorable premorbid status. Applying the liberal and restrictive eligibility criteria to our study population, 33 and 5 patients, respectively, would have been candidates for ECPR (Supplemental Online Appendix C). This translates to an estimated $0.2-1.4$ patients per month that would have potentially qualified for ECPR at our institution.

\section{DISCUSSION}

This study was designed to evaluate the feasibility of ECPR at our institution by first assessing the existing 
volume of out-of-hospital cardiac arrest patients. In our center, 3 to $18 \%$ of out-of-hospital cardiac arrest patients brought to our ED per year appear to meet criteria for ECPR, depending on the strictness of inclusion criteria that our center may choose to implement. Our findings suggest that a target population for this novel therapy does exist in our region, although it remains small. These data may help illustrate an anticipated volume for similar medium-sized academic centers.

Limited comparative data exist for these rates, although our estimated numbers (5-33 patients out of an out-of-hospital cardiac arrest pool of 187 patients) are aligned with the registry study by Grunau et al., ${ }^{10}$ which found that approximately $10 \%$ of prehospital out-of-hospital cardiac arrest patients would be eligible for ECPR based on their selection criteria. In planning implementation at our center, we expect that it will be prudent to adopt somewhat more restrictive inclusion criteria (Figure 1) initially, to optimize systems and processes, and foster institutional buy-in.

The main limitation to our study was the quality of records used. As the study was retrospective, the largely handwritten records reviewed had, at times, missing or incongruous variables that were of interest to our study. As such, the true number of eligible ECPR candidates might be underestimated. Furthermore, as a singleinstitution study, our data do not include potential candidates within a reasonable geographic radius who were brought to community hospitals. The sample population may also be biased by prehospital termination-of-resuscitation (TOR) criteria used in conjunction with on-line medical control: either biasing toward patients with a higher potential survivability (those not terminated in the field), or by increasing "lowflow" times for patients who may have otherwise been good ECPR candidates. This highlights a need for system-level protocols to be developed, such as the load-and-go criteria. Finally, our study is limited by the retrospective application of criteria, which may prove more difficult to ascertain prospectively for an unknown patient coming into the ED in arrest.

\section{CONCLUSION}

We found that $6-40 \%$ of refractory out-of-hospital cardiac arrest patients presenting to a tertiary care ED would have been potential ECPR candidates, translating to $0.2-1.4$ patients per month. A majority of patients brought to our EDs in cardiac arrest had at least some favourable characteristics. These findings suggest that there appears to a distinct, although small, population of cardiac arrest patients that would be eligible for an ED ECPR program at our institution.

Supplemental material: The supplemental material for this article can be found at https://doi.org/10.1017/cem.2019.472.

Competing interests: None declared.

\section{REFERENCES}

1. Brooks SC, Schmicker RH, Rea TD, et al. Out-of-hospital cardiac arrest frequency and survival: evidence for temporal variability. Resuscitation 2010;81(2):175-81.

2. Chan PS, McNally B, Tang F, Kellermann A; CARES Surveillance Group. Recent trends in survival from out-of-hospital cardiac arrest in the United States. Circulation 2014;130(21):1876-82.

3. Brooks SC, Shemie SD, Torrance S, et al. Barriers and opportunities related to extracorporeal cardiopulmonary resuscitation for out-of-hospital cardiac arrest in Canada: a report from the first meeting of the Canadian ECPR Research Working Group. C7EM 2018;20(4):507-17.

4. Bellezzo JM, Shinar Z, Davis DP, et al. Emergency physician-initiated extracorporeal cardiopulmonary resuscitation. Resuscitation 2012;83:966-70.

5. Yannopoulos D, Bartos JA, Martin C, et al. Minnesota Resuscitation Consortium's Advanced Perfusion and Reperfusion Cardiac Life Support Strategy for Out-of-Hospital Refractory Ventricular Fibrillation. $\mathcal{f}$ Am Heart Assoc 2016; 5(6): 0003732 .

6. Stub D, Bernard S, Pellegrino V, et al. Refractory cardiac arrest treated with mechanical CPR, hypothermia, ECMO and early reperfusion (the CHEER trial). Resuscitation 2015;86:88-94.

7. Johnson NJ, Acker M, Hsu CH, et al. Extracorporeal life support as rescue strategy for out-of-hospital and emergency department cardiac arrest. Resuscitation 2014;85:1527-32.

8. Muller G, Flecher E, Lebreton G, et al. The ENCOURAGE mortality risk score and analysis of long-term outcomes after VA-ECMO for acute myocardial infarction with cardiogenic shock. Intensive Care Med 2016;42(3): 370-8.

9. Sakamoto T, Morimura N, Nagao K, et al. Extracorporeal cardiopulmonary resuscitation versus conventional cardiopulmonary resuscitation in adults with out-of-hospital cardiac arrest: a prospective observational study. Resuscitation 2014;85:762-8.

10. Grunau B, Scheuermeyer FX, Stub D, et al. Potential candidates for a structured Canadian ECPR program for out-of-hospital cardiac arrest. CFEM 2016;18(6):453-60. 